\title{
TAVI in Patients with Mitral Annular Calcification and/or Mitral Stenosis
}

\author{
Andreas Schaefer ${ }^{1}$ Harun Sarwari ${ }^{1} \quad$ Niklas Schofer $^{2} \quad$ Yvonne Schneeberger $^{1}$ Dirk Westermann ${ }^{2}$ \\ Gerhard Schoen ${ }^{3}$ Stefan Blankenberg ${ }^{2}$ Hermann Reichenspurner ${ }^{1}$ Ulrich Schäfer ${ }^{2}$ Lenard Conradi $^{1}$ \\ 1 Department of Cardiovascular Surgery, University Heart Center \\ Hamburg, Hamburg, Germany \\ 2 Department of General and Interventional Cardiology, University \\ Heart Center Hamburg, Hamburg, Germany \\ ${ }^{3}$ Department of Medical Biometry and Epidemiology, University \\ Medical Center Hamburg-Eppendorf, Hamburg, Germany \\ Thorac Cardiovasc Surg 2021;69:428-436. \\ Address for correspondence Andreas Schaefer, MD, MHBA, \\ Department of Cardiovascular Surgery, University Heart Center \\ Hamburg, Martinistraße 52, Hamburg 20246, Germany \\ (e-mail: and.schaefer@uke.de).
}

\begin{abstract}
Keywords

- transcatheter aortic valve implantation

- mitral stenosis

- aortic valve

- mitral valve

- aortic valve stenosis

Background We herein aimed for analysis of influence of mitral annular calcification (MAC) and mitral stenosis (MS) on outcomes in transcatheter aortic valve implantation (TAVI). Methods Between 11/2009 and 06/2017, 1,058 patients underwent TAVI in the presence of concomitant MAC or MS at our center. Subgroups were built and multivariate logistic regression, COX regression, Kaplan-Meier survival analyses, and receiver operating characteristics method were performed.

Results Thirty-day mortality was $7.5 \%(79 / 1,058)$ with highest mortality in patients severe MS (MAC: $3.4 \%$ vs. mild MS: $5.9 \%$ vs. moderate MS: $15.0 \%$ vs. severe MS: $72.7 \%$; $p<0.001$ ). Moderate-to-severe MS (odds ratio [OR]: 7.75, confidence interval [Cl]: 3.94-16.26, $p<0.001$ ), impaired left ventricular ejection fraction (OR: $1.38, \mathrm{Cl}: 1.10-1.72$, $p<0.01$ ), and coronary artery disease (OR: $1.36, \mathrm{Cl}: 1.11-1.67, p<0.01)$ were predictive of 30-day survival. Left ventricular systolic/end-diastolic pressure drop of $<59.5 \mathrm{~mm} \mathrm{Hg} \mathrm{/}$ $<19.5 \mathrm{~mm} \mathrm{Hg}$ was associated with increased mortality.

Conclusions TAVI in the presence of MAC and mild MS is associated with acceptable acute outcomes but should be considered high-risk procedures in patients with moderate and especially those with severe MS. Our results suggest adverse hemodynamics after TAVI with concomitant MS, which may be caused by underfilling of the left ventricle leading to low-cardiac output.
\end{abstract}

\section{Introduction}

Transcatheter aortic valve implantation (TAVI) is an established therapy for severe aortic valve stenosis in high-risk patients. ${ }^{1,2}$ Recent evidence suggests also benefit for intermediate-risk patients compared with surgical aortic valve replacement. ${ }^{3,4}$ Risk factors for impaired outcomes subsequent to TAVI have been described extensively. Besides common determinants for postoperative morbidity and mortality such as age, gender, frailty, or chronic kidney disease, ${ }^{5-8}$ procedure-specific risk factors for particular clinical outcomes have been described.
These include anatomical, electrocardiogram- and valve-platform-related determinants for postinterventional permanent pacemaker (PPM) implantation ${ }^{9}$ or landing zone calcification patterns for prediction of significant paravalvular leakage $(\mathrm{PVL})$ after TAVI. ${ }^{10}$

Recently, mitral annular calcification (MAC) was shown to be predictive of increased all-cause and cardiovascular mortality as well as conduction abnormalities post-TAVI. ${ }^{11}$ Depending on the definition, 17 to $50 \%$ of patients evaluated for TAVI exhibit significant MAC in preoperative echocardiography and/or computed tomography. ${ }^{11,12}$ MAC as expression received

August 10, 2019

accepted after revision

November 19, 2019

published online

March 1, 2020 (c) 2020. Thieme. All rights reserved.

Georg Thieme Verlag KG,

Rüdigerstraße 14,

70469 Stuttgart, Germany
DOI https://doi.org/ 10.1055/s-0039-1700966. ISSN 0171-6425. 
of atherosclerosis can also extend into the mitral leaflets and cause mitral valve stenosis (MS), which is the case in 1 to $2 \%$ of patients with MAC. ${ }^{13}$ Little is known about the influence of significant MS on outcomes in TAVI procedures. However, isolated TAVI for aortic stenosis (AS) in patients with MS may lead to postinterventional hemodynamic compromise due to acute decompression of a usually stiff, small, and hypertrophied left ventricle (LV) with MS induced persistently lowered filling volumes. Furthermore, continued postinterventional presence of postcapillary pulmonary hypertension may lead to hemodynamic impairment. ${ }^{14}$

We herein investigate outcomes of patients with AS and concomitant MAC and/or MS receiving TAVI focusing on acute hemodynamic changes and outcome differences between severity levels of MS.

\section{Materials and Methods}

\section{Patients}

Between 11/2009 and 06/2017, 2,582 patients were treated by TAVI at our center. Of those 1,058 patients ( $40.9 \%$; female $54.8 \%$, $80.8 \pm 6.8$ years, logistic European System for Cardiac Operative Risk Evaluation [logEUROScore] I $19.1 \pm 13.2$ ) underwent TAVI for severe symptomatic AS in the presence of concomitant MAC or MS. Subgroups were built including 352/1,058 (33.3\%) patients with MAC, 528/1,058 (49.9\%) patients with mild MS, $168 / 1,058$ (15.9\%) patients with moderate MS, and 11/1,058 (1.0\%) patients with severe MS.

Allocation of patients to TAVI followed current international recommendations. ${ }^{1}$ Patient data and follow-up were retrieved from our dedicated hospital TAVI database. All patients suffered from AS and concomitant MAC and/or MS as determined by echocardiography. MS was classified by mean pressure gradient over the MV (mean pressure gradient 2 to $5 \mathrm{~mm} \mathrm{Hg}$ : mild MS; 5 to $10 \mathrm{~mm} \mathrm{Hg}$ : moderate MS; 10 to $15 \mathrm{~mm} \mathrm{Hg}$ : severe MS). ${ }^{15}$ MAC was visualized by echocardiography and/or multislice computed tomography. Written informed consent was obtained from all patients prior to the procedure.

\section{Diagnostic Workup and Study Procedure}

Diagnostics and procedures followed institutional routine as previously described. ${ }^{9}$ All procedures were accompanied by pre- and postinterventional invasive simultaneous pressure measurements of the LV and Aorta (AO) for the determination of periinterventional hemodynamic course (peak/mean pressure gradient, LV pressure systolic/end diastolic, AO pressure systolic/diastolic, $\Delta p$ LV pre- and postinterventional $/ \Delta p$ AO pre- and postinterventional). Transcatheter heart valve (THV) function was subsequently assessed by transesophageal echocardiography (TEE) and aortic root angiography.

\section{Statistics}

Baseline, intraprocedural, and acute follow-up data up to 30 days were prospectively collected and entered into a dedicated standardized database and retrospectively analyzed. Clinical endpoints were adjudicated in accordance with the updated standardized Valve Academic Research Consortium (VARC-2) definitions. ${ }^{16}$ Data are presented as absolute numbers and percentages for categorical variables and mean values and standard deviation for continuous variables unless stated otherwise. For determination of independent risk factors for 30-day mortality multivariate logistic regression was performed including odds ratios and confidence intervals (CI). Survival in follow-up was investigated utilizing Kaplan-Meier survival analysis and COX regression comprising hazard ratios (HRs) and CI. For the determination of significant hemodynamic thresholds for postoperative mortality receiver operating characteristic for simultaneous $\mathrm{LV} / \mathrm{AO}$ pressure measurements ( $\Delta \mathrm{LV}$ systolic, $\Delta \mathrm{LV}$ end diastolic, $\Delta \mathrm{AO}$ systolic, $\Delta$ AO diastolic) were performed.

\section{Results}

\section{Baseline Demographics}

Baseline demographics revealed a symptomatic and comorbid study population with a mean log EuroSCORE I of $20.7 \pm 14.6$ and New York Heart Association $\geq$ III (NYHA $\geq$ III) in $84.3 \%$ of patients. Significant difference between subgroups was found regarding gender distribution.

Detailed patient demographics of the entire study population and subgroups are summarized in -Table 1.

\section{Periprocedural Data}

Transthoracic echocardiography (TTE) and TEE at baseline confirmed severe AS in all subgroups. Mitral regurgitation (MR) $\geq$ grade II+ was most frequent in patients with moderate MS without reaching statistical significance. Fluoroscopy time was prolonged in patients with moderate and severe MS.

Transfemoral (TF) approach was utilized in $64.4 \%$ $(681 / 1058)$ of the entire study cohort and transapical (TA) approach in $35.6 \%$ (376/1058) without differences between the subgroups. Balloon-expandable THV were most frequently utilized for TAVI in patients with MAC. Conversely, self- and mechanical-expandable THV were most frequently used in patients with significant MS. Baseline echocardiography and periprocedural data are summarized in - Table 2.

\section{Echocardiographic and Acute Clinical Outcome Data}

Peak and mean aortic transvalvular gradients of the entire study cohort as determined by TTE prior to discharge decreased from $61.7 \pm 26.4$ to $19.9 \pm 9.2 \mathrm{~mm} \mathrm{Hg}$ and $35.8 \pm 16.5$ to $10.1 \pm 4.9 \mathrm{~mm} \mathrm{Hg}$ (both $p<0.01$ ), respectively. Postinterventionally, PVL $\geq$ moderate was found in $4.1 \%$ (40/1058) of the patients.

Device success and early safety according to VARC-2 definitions were achieved in 90.6\% (933/1058) and $81.2 \%$ $(837 / 1058)$ of the patients with lowest device success in patients with MAC and lowest early safety rates in patients with moderate and severe MS. All-cause mortality was 7.5\% (79/1058) in all patients with highest rates in patients with moderate and severe MS (MAC: $3.4 \%$, mild MS: $5.9 \%$, moderate MS 15.0\%, severe MS: 72.7\%; $p<0.001)$. Regarding stroke, myocardial infarction, major bleeding, and PPM implantation 
Table 1 Baseline demographics for entire study population and subgroups

\begin{tabular}{|c|c|c|c|c|c|c|}
\hline & $\begin{array}{l}\Sigma \\
n=1058\end{array}$ & $\begin{array}{l}\text { MAC } \\
n=352\end{array}$ & $\begin{array}{l}\text { Mild MS } \\
n=528\end{array}$ & $\begin{array}{l}\text { Moderate MS } \\
n=167\end{array}$ & $\begin{array}{l}\text { Severe } \\
n=11\end{array}$ & $p$-Value ${ }^{a}$ \\
\hline Age, years & $80.0 \pm 6.8$ & $80.6 \pm 7.0$ & $81.1 \pm 6.7$ & $80.2 \pm 6.9$ & $82.0 \pm 5.2$ & 0.40 \\
\hline Female gender, \% (n) & $54.8(580)$ & $52.3(184)$ & $52.7(278)$ & $67.1(112)$ & $54.5(6)$ & $<0.01$ \\
\hline BMI, $\mathrm{kg} / \mathrm{m}^{2}$ & $26.8 \pm 7.7$ & $26.3 \pm 8.4$ & $26.9 \pm 7.8$ & $27.5 \pm 5.4$ & $28.8 \pm 7.5$ & 0.26 \\
\hline logEuroSCORE I, \% & $20.7 \pm 14.6$ & $19.1 \pm 12.2$ & $18.9 \pm 13.7$ & $19.0 \pm 13.2$ & $25.9 \pm 19.5$ & 0.15 \\
\hline Diabetes, \% (n) & $29.8(315)$ & $28.7(101)$ & $29.9(158)$ & $32.3(54)$ & $18.2(2)$ & 0.69 \\
\hline Arterial hypertension, \% ( $n$ ) & $84.0(889)$ & $82.4(290)$ & $85.4(451)$ & $82.6(138)$ & $90.9(10)$ & 0.55 \\
\hline Stroke, \% (n) & $15.8(167)$ & $13.4(47)$ & $16.7(88)$ & $17.4(29)$ & $27.3(3)$ & 0.35 \\
\hline Coronary artery disease, $\%(n)$ & $61.5(649)$ & $61.1(214)$ & $63.2(334)$ & $54.8(91)$ & $90.9(10)$ & 0.18 \\
\hline Previous sternotomy, \% (n) & $15.4(163)$ & $16.8(59)$ & $14.9(79)$ & $12.6(21)$ & $36.3(4)$ & 0.15 \\
\hline Extracardiac arteriopathy, ${ }^{\mathrm{b}} \%(n)$ & $28.4(301)$ & $32.1(113)$ & $25.6(135)$ & $29.3(49)$ & $36.4(4)$ & 0.18 \\
\hline Arrhythmia, \% (n) & $30.5(312)$ & $32.7(113)$ & $30.0(152)$ & $27.3(44)$ & $30.0(3)$ & 0.18 \\
\hline Permanent pacemaker, \% (n) & $6.8(70)$ & $5.8(20)$ & $6.1(31)$ & $11.8(19)$ & $0.0(0)$ & 0.06 \\
\hline COPD $^{\mathrm{b}}>$ Gold II, \% (n) & $15.4(163)$ & $16.8(59)$ & $15.0(79)$ & $12.6(21)$ & $36.4(4)$ & 0.15 \\
\hline Creatinine, $\mathrm{mg} / \mathrm{dL}$ & $1.4 \pm 1.3$ & $1.5 \pm 1.3$ & $1.4 \pm 1.3$ & $1.4 \pm 1.1$ & $1.4 \pm 0.4$ & 0.77 \\
\hline Pulmonary hypertension $^{\mathrm{b}}>55 \mathrm{~mm} \mathrm{Hg}, \%(n)$ & $41.9(444)$ & $41.4(146)$ & $42.4(224)$ & $40.1(67)$ & $63.6(7)$ & 0.32 \\
\hline LVEF $\leq 45 \%, \%(n)$ & $20.9(222)$ & $21.6(76)$ & $21.2(112)$ & $18.6(31)$ & $27.7(3)$ & 0.81 \\
\hline $\mathrm{NYHA} \geq \mathrm{III}, \%(n)$ & $84.3(892)$ & $83.2(293)$ & $85.9(454)$ & 82.0 (137) & $72.7(8)$ & 0.36 \\
\hline
\end{tabular}

Abbreviations: ANOVA, analysis of variance; BMI, body mass index; COPD, chronic obstructive pulmonary disease; logEuroSCORE, logistic European System for Cardiac Operative Risk Evaluation; LVEF, left ventricular ejection fraction; MAC, mitral annular calcification; MS, mitral stenosis; NYHA, New York Heart Association.

${ }^{a}$ ANOVA for metric variables and $\mathrm{chi}^{2}$ for categorial variables.

${ }^{b}$ Extracardiac arteriopathy, COPD and pulmonary hypertension according to EuroSCORE definitions.

rates no significant differences were found between the subdivided patient cohorts. Access site complications were most frequent in patients with mild and severe MS.

Echocardiographic and detailed acute clinical outcome data are summarized in - Table 3.

In multivariate logistic regression for 30-day survival, including 17 variables (see $\mathbf{-}$ Supplementary Table $\mathbf{1}$ ), independent risk factors for postoperative mortality were moderate/severe MS, reduced LV ejection fraction at baseline, and coronary artery disease (see $\boldsymbol{-}$ Fig. $\mathbf{1}$ ).

When comparing 30-day mortality of TA and TF subgroups, no significant differences were found. However, when comparing MAC/MS subgroups, patients with moderate and severe MS provided with TA-TAVI presented a significantly higher overall acute mortality compared with the TF approach (see - Fig. 2).

\section{Invasive Hemodynamic Measurements during TAVI}

Pressure changes ( $\Delta p \mathrm{LV}$ systolic/end-diastolic, $\Delta \mathrm{p}$ AO systolic/ diastolic) were calculated for every patient. Here, significant differences were found between the subgroups with highest LV and aortic pressure drop (absolute difference between LV and aortic systolic and (end) diastolic pressure before and after THV implantation in simultaneous measurements) in patients with moderate and severe MS. For determination of threshold values for LV pressure decline after TAVI, receiver operating characteristic (ROC) analyses were performed. Here, a $\Delta p$ LV systolic of $59.5 \mathrm{~mm} \mathrm{Hg}$ and a $\Delta p$ LV end-diastolic of $19.5 \mathrm{~mm} \mathrm{Hg}$ were shown to be thresholds for an increased acute mortality (see - Fig. 3).

Hemodynamics of the entire study population and subgroups are summarized in -Table 4.

\section{Follow-Up Analysis}

Follow-up was completed in $92.3 \%$ of cases with a mean follow-up time of 703.5 days. Kaplan-Meier survival analysis presented lowest long-term survival in patients with severe MS and no significant differences of patients with MAC, mild, and moderate MS (see - Fig. 4).

COX regression for influence of severity of MS and approach on long-term mortality presented a HR of 12.98 (2.5\%: 6.31, 97.5\%:26.70; $p<0.001)$ for severe MS and a HR of 1.75 (2.5\%: 1.44, 97.5\%:2.13) for TF approach $(p<0.001)$.

\section{Discussion}

\section{Main Findings}

Main findings of the herein conducted study are (1) MAC and/or MS are a frequent findings in patients undergoing TAVI for severe AS, affecting over one third of all patients treated at our center in a 9-year time interval; (2) TAVI is associated with acceptable acute outcomes in patients with MAC and mild MS, and should be considered high-risk procedures in patients with moderate and severe MS due to significantly increased early mortality rates; (3) moderate/severe MS is an independent risk factor for acute mortality after TAVI; (4) evaluation of 
Table 2 Periprocedural data for entire study population and subgroups

\begin{tabular}{|c|c|c|c|c|c|c|}
\hline & $\begin{array}{l}\Sigma \\
n=1058\end{array}$ & $\begin{array}{l}\text { MAC } \\
n=352\end{array}$ & $\begin{array}{l}\text { Mild MS } \\
n=528\end{array}$ & $\begin{array}{l}\text { Moderate MS } \\
n=167\end{array}$ & $\begin{array}{l}\text { Severe MS } \\
n=11\end{array}$ & $p$-Value ${ }^{a}$ \\
\hline Baseline EOA, $\mathrm{cm}^{2}(\mathrm{AV})$ & $0.7 \pm 0.2$ & $0.8 \pm 0.3$ & $0.8 \pm 0.2$ & $0.7 \pm 0.2$ & $0.8 \pm 0.3$ & 0.40 \\
\hline Peak gradient, mm Hg (AV) & $61.7 \pm 26.4$ & $60.5 \pm 27.1$ & $61.1 \pm 25.4$ & $66.2 \pm 27.0$ & $58.2 \pm 36.0$ & 0.10 \\
\hline Mean gradient, mm Hg (AV) & $35.8 \pm 16.5$ & $34.7 \pm 16.6$ & $35.4 \pm 15.7$ & $39.2 \pm 17.7$ & $35.8 \pm 24.6$ & 0.02 \\
\hline Peak gradient, mm Hg (MV) & $9.9 \pm 4.1$ & $5.4 \pm 1.6$ & $8.7 \pm 3.0$ & $14.9 \pm 3.6$ & $18.9 \pm 3.8$ & $<0.001$ \\
\hline Mean gradient, mm Hg (MV) & $3.5 \pm 1.7$ & $1.8 \pm 0.7$ & $2.9 \pm 0.9$ & $5.9 \pm 1.1$ & $18.8 \pm 3.7$ & $<0.001$ \\
\hline MR $\geq$ grade $I I+, \%(n)$ & $30.8(326)$ & $34.0(120)$ & $27.3(144)$ & $35.3(59)$ & $27.3(3)$ & 0.09 \\
\hline Procedure time, ${ }^{b}$ min & $92.8 \pm 47.4$ & $89.8 \pm 46.9$ & $93.6 \pm 44.6$ & $94.9 \pm 53.8$ & $118.6 \pm 75.7$ & 0.16 \\
\hline Fluoroscopy time, min & $16.3 \pm 16.5$ & $13.4 \pm 9.9$ & $17.8 \pm 19.7$ & $18.0 \pm 16.0$ & $20.3 \pm 14.6$ & $<0.001$ \\
\hline Contrast agent, $\mathrm{mL}$ & $169.8 \pm 109.1$ & $170.8 \pm 141.3$ & $169.7 \pm 91.2$ & $166.5 \pm 74.1$ & $196.3 \pm 147.5$ & 0.84 \\
\hline \multicolumn{7}{|l|}{ Access, \% (n) } \\
\hline TF & $64.4(681)$ & $59.7(210)$ & $66.7(352)$ & $67.7(113)$ & $60.0(6)$ & 0.14 \\
\hline TA & $35.6(376)$ & $40.3(142)$ & $33.3(176)$ & $32.3(54)$ & $40.0(4)$ & 0.14 \\
\hline \multicolumn{7}{|l|}{ Valve type, \% (n) } \\
\hline Sapien XT/3 & $52.8(559)$ & $63.9(225)$ & $46.6(246)$ & $50.9(85)$ & $27.3(3)$ & $<0.001$ \\
\hline Acurate TA/neo-TF & $10.6(112)$ & $5.9(21)$ & $12.3(65)$ & $13.7(23)$ & $27.3(3)$ & $<0.01$ \\
\hline CoreValve & $8.2(87)$ & $9.4(33)$ & $7.7(41)$ & $7.8(13)$ & 1 & 0.85 \\
\hline BioValve & $0.8(8)$ & $0.6(2)$ & $0.8(4)$ & $1.2(2)$ & 1 & 0.02 \\
\hline Engager & $5.9(63)$ & $5.9(21)$ & $5.7(30)$ & $5.9(10)$ & $18.2(2)$ & 0.39 \\
\hline JenaValve & $8.9(95)$ & $9.6(34)$ & $9.8(52)$ & $5.4(9)$ & 1 & 0.35 \\
\hline Lotus & $7.3(77)$ & $2.8(10)$ & $9.6(51)$ & $8.4(14)$ & $18.2(2)$ & $<0.001$ \\
\hline Portico & $5.4(57)$ & $1.7(6)$ & $7.7(41)$ & $5.4(9)$ & $9.1(1)$ & $<0.01$ \\
\hline Predilatation, \% (n) & $74.0(781)$ & $74.1(261)$ & $73.0(384)$ & $76.5(127)$ & $81.8(9)$ & 0.76 \\
\hline Postdilatation, \% (n) & $21.2(223)$ & $17.9(63)$ & 22.7 (119) & $22.3(37)$ & $36.4(4)$ & 0.20 \\
\hline
\end{tabular}

Abbreviations: ANOVA, analysis of variance; AV, aortic valve; EOA, effective orifice area; LVOT, left ventricular outflow tract; MAC, mitral annular calcification; MR, mitral regurgitation; MS, mitral stenosis; MSCT, multislice computed tomography; MV, mitral valve; TA, transapical; TF, transfemoral.

${ }^{\mathrm{a}}$ ANOVA for metric variables and $\mathrm{chi}^{2}$ for categorial variables.

${ }^{\mathrm{b}}$ Median values to account for outliers.

pre- and postoperative hemodynamics suggests increased LV pressure drop after TAVI as possible reason for impaired outcomes in the study cohort; (5) TA approach is adversely impacting periinterventional results in patients with moderate and severe MS, but is not an independent risk factor for acute mortality.

MAC as risk factor for adverse clinical results in TAVI procedures was recently described. ${ }^{11}$ In this work of Abramowitz et al, severe MAC was shown to be an independent risk factor for cardiovascular mortality subsequent to TAVI with a HR of 2.35. It was also shown to be predictive of postinterventional PPM implantation with an odds ratio of 2.83. In our study, patients with MAC had comparable outcomes with an overall mortality of $3.4 \%$ and a PPM implantation rate of $21.1 \%$. Nevertheless, these are clearly higher rates compared with our general TAVI cohort. ${ }^{9,17}$ Since we did not perform analysis of MAC severity, our results may be not directly comparable to this prior work.

A current analysis of the Society of Thoracic Surgeons/ American College of Cardiology Transcatheter Valve Therapies
Registry found MS in one-tenth of patients undergoing TAVI with severe MS as independent risk factor for 1-year mortality. ${ }^{18}$ In this study patients with severe MS experienced an inhospital mortality of 5.6\%. Our analysis revealed markedly higher mortality rates of patients with moderate/severe MS. Reasons for this discrepancy are most likely multifactorial. First, the two cited studies are so far the only analyses regarding influence of MAC and MS on outcomes in TAVI procedures. Therefore, general conclusions regarding impact of this echocardiography finding/comorbidity in TAVI should be made with caution. Second, diagnosis modalities differed between our study, in which MAC/MS was partly diagnosed by TTE/TEE, and previous works, in which only multislice computed tomography was utilized to detect MAC/MS. Third, patients undergoing TAVI in the presence of concomitant MS in this study presented with a particularly pronounced risk profile. This fact is reflected by the high proportion of patients with pulmonary hypertension, extracardiac arteriopathy, concomitant MS itself and patients receiving TAVI via TA access, not representing the current distribution of TF (95\%) and TA (4\%) approaches in 
Table 3 Clinical outcome and echocardiographic results at discharge for entire study population and subgroups

\begin{tabular}{|c|c|c|c|c|c|c|}
\hline & $\begin{array}{l}\Sigma \\
n=1058\end{array}$ & $\begin{array}{l}\text { MAC } \\
n=352\end{array}$ & $\begin{array}{l}\text { Mild MS } \\
n=528\end{array}$ & $\begin{array}{l}\text { Moderate MS } \\
n=167\end{array}$ & $\begin{array}{l}\text { Severe MS } \\
n=11\end{array}$ & $p$-Value ${ }^{a}$ \\
\hline All-cause mortality (30 days), \% ( $n$ ) & $7.5(79)$ & $3.4(12)$ & $5.9(31)$ & $15.0(25)$ & $72.7(8)$ & $<0.001$ \\
\hline Cardiovascular or unknown, \% (n) & $7.5(79)$ & $3.4(12)$ & $5.9(31)$ & $15.0(25)$ & $72.7(8)$ & $<0.001$ \\
\hline Stroke (disabling), \% (n) & $6.2(64)$ & $4.3(15)$ & $7.4(38)$ & $6.2(10)$ & $9.1(1)$ & 0.32 \\
\hline Myocardial infarction, \% (n) & $1.0(10)$ & $1.2(4)$ & $0.8(4)$ & $1.2(2)$ & $0.0(0)$ & 0.91 \\
\hline Bleeding (major/life threatening), \% ( $n$ ) & $18.9(194)$ & $16.8(58)$ & $21.2(109)$ & $15.0(24)$ & $27.3(3)$ & 0.18 \\
\hline $\begin{array}{l}\text { Access site complications } \\
\text { (minor/major), \% ( } n)\end{array}$ & $19.8(204)$ & $15.6(54)$ & $23.3(120)$ & $16.9(27)$ & $27.3(3)$ & 0.03 \\
\hline Acute kidney injury (AKIN 2, 3), \% (n) & $24.4(251)$ & $25.5(88)$ & $23.7(122)$ & $23.8(38)$ & $27.3(3)$ & 0.93 \\
\hline Pacemaker implantation, \% (n) & $21.5(222)$ & $21.1(73)$ & $20.8(107)$ & $26.2(42)$ & $0.0(0)$ & 0.15 \\
\hline Device success, ${ }^{\mathrm{b}} \%(n)$ & $90.6(933)$ & $87.0(301)$ & $93.2(478)$ & $90.0(144)$ & $90.9(10)$ & 0.03 \\
\hline Early safety, ${ }^{\mathrm{b}} \%(n)$ & $81.2(837)$ & $83.2(288)$ & $81.1(417)$ & $80.0(128)$ & $27.3(3)$ & $<0.01$ \\
\hline Intensive care unit stay, days & $2.5 \pm 3.5$ & $2.6 \pm 4.3$ & $2.4 \pm 2.8$ & $2.6 \pm 3.8$ & $2.8 \pm 2.8$ & 0.82 \\
\hline Hospital stay, days & $11.2 \pm 8.7$ & $11.3 \pm 9.9$ & $11.2 \pm 8.2$ & $10.9 \pm 7.2$ & $10.0 \pm 6.1$ & 0.95 \\
\hline $\mathrm{EOA}, \mathrm{cm}^{2}$ & $1.7 \pm 0.4$ & $1.7 \pm 0.4$ & $1.7 \pm 0.4$ & $1.6 \pm 0.3$ & $1.4 \pm 0.2$ & 0.6 \\
\hline Peak gradient, mm Hg (AV) & $19.9 \pm 9.2$ & $19.3 \pm 8.7$ & $20.2 \pm 9.3$ & $20.0 \pm 9.5$ & $28.0 \pm 15.2$ & 0.12 \\
\hline Mean gradient, mm Hg (AV) & $10.1 \pm 4.9$ & $9.7 \pm 4.5$ & $10.3 \pm 5.0$ & $10.2 \pm 5.0$ & $15.4 \pm 9.8$ & 0.03 \\
\hline Paravalvular leakage $\geq$ grade II, \% (n) & $4.1(40)$ & $5.3(17)$ & $3.3(16)$ & $4.7(7)$ & $0.0(0)$ & 0.50 \\
\hline
\end{tabular}

Abbreviations: AKIN, Acute Kidney Injury Network; AV, aortic valve; EOA, effective orifice area; MAC, mitral annular calcification; MS, mitral stenosis; MV, mitral valve; VARC, Valve Academic Research Consortium.

${ }^{a}$ ANOVA for metric variables and $\mathrm{chi}^{2}$ for categorial variables.

${ }^{b}$ According to VARC-2 definitions.

our center and the latest literature. ${ }^{19}$ Most importantly, the cited studies investigated time intervals of 3 to 4 years, whereas our patient cohort comprises a 9-year experience.

Also, the high rates of preoperative PPM, especially in the cohort with moderate MS, and the increased utilization of selfexpandable THV, which are more likely to be used in patients with a calcified left ventricular outflow tract, indicate a treated patient population with an extensively increased cardiovascular calcium load, especially when taking into consideration that MAC is an expression of an ubiquitary cardiovascular calcification process. $^{20}$

TA access was described before as independent risk factor for impaired outcomes subsequent to TAVI. ${ }^{21}$ Commonly, lower invasiveness of the TF approach and a more comorbid patient population undergoing TA TAVI are considered to be main reasons for inferior outcomes of TATAVI. Impact of the TA approach on mortality rates of patients with moderate and severe MS in this study is in line with prior studies describing

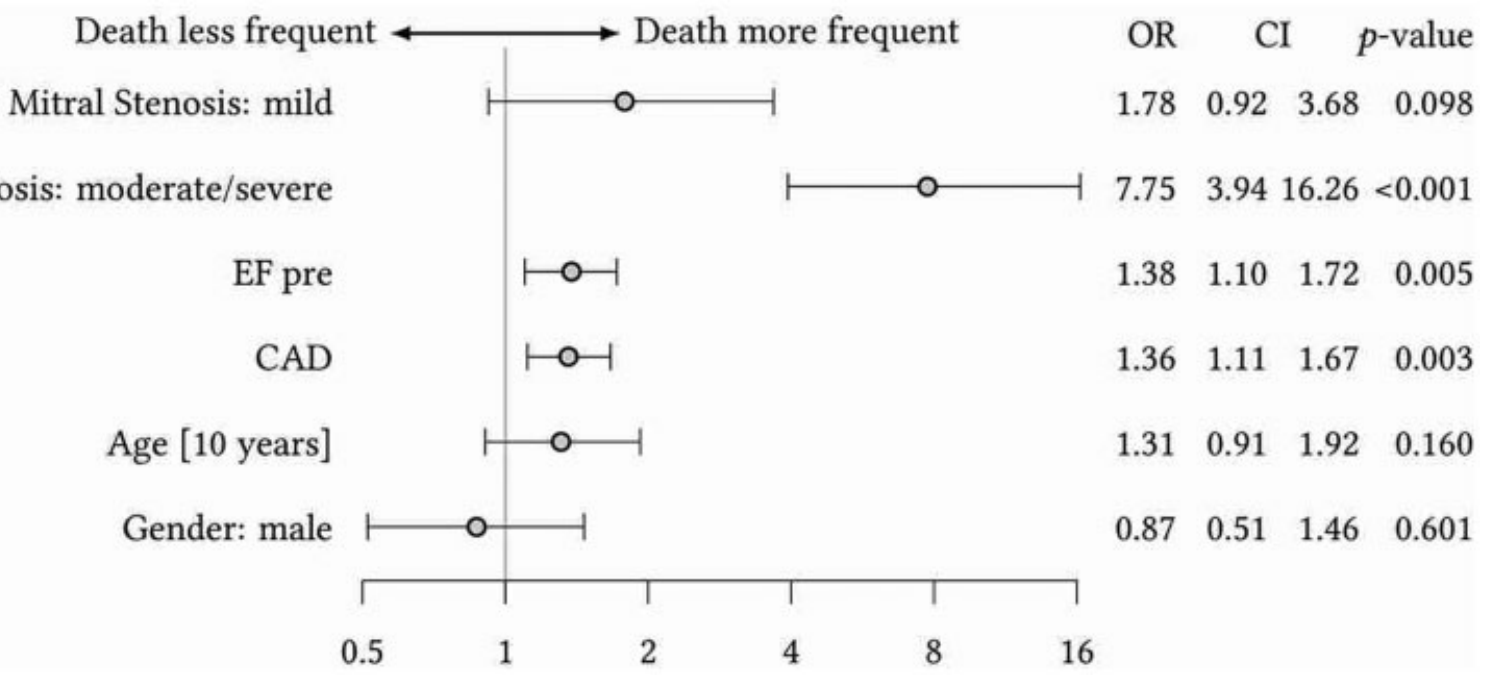

Fig. 1 Final model of multivariate logistic regression for 30-day survival. CAD, coronary artery disease; $\mathrm{Cl}$, confidence interval; EF, ejection fraction; OR, odds ratio. 


\section{Entire patient cohort $(n=1058)$}

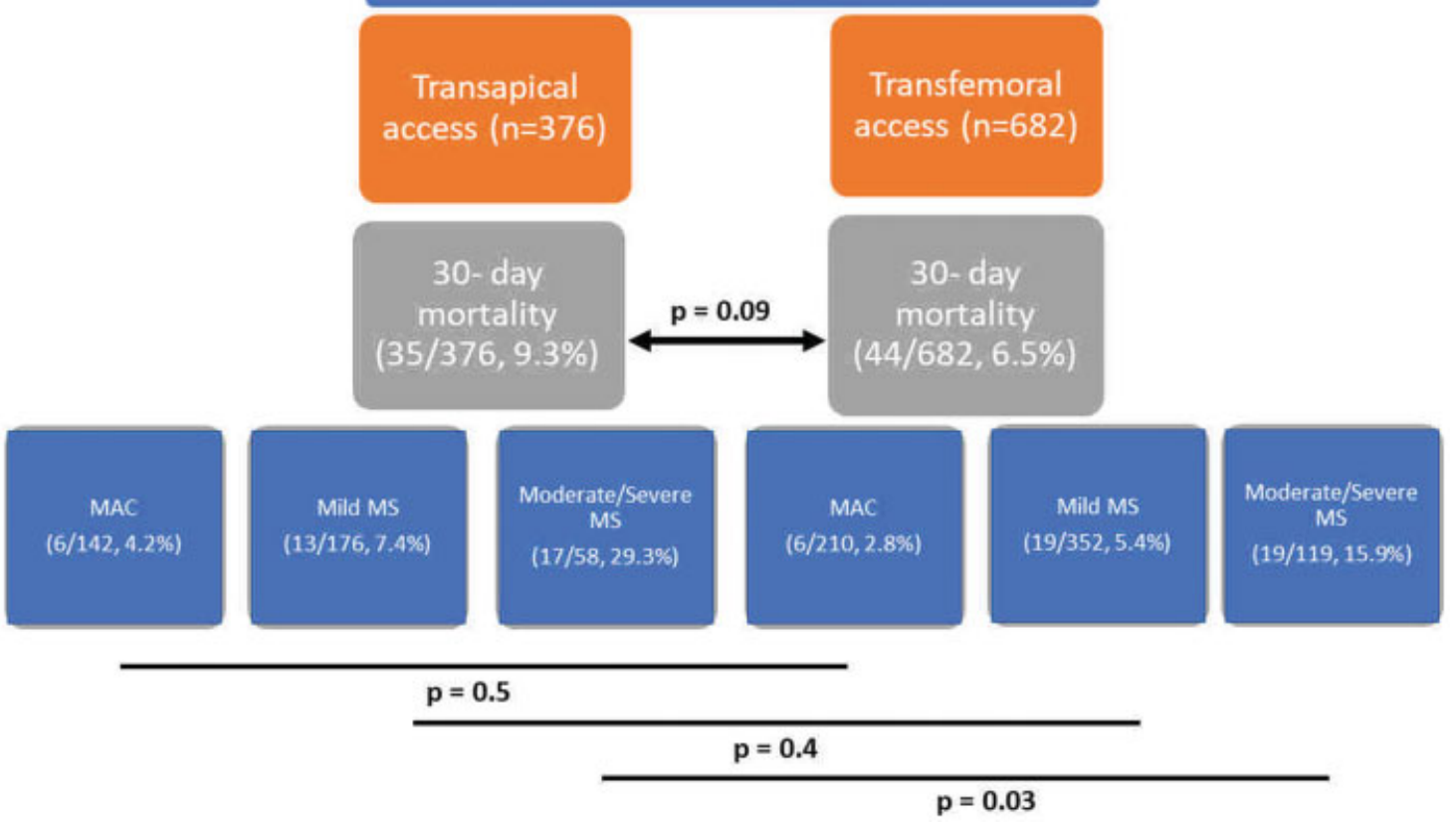

Fig. 2 Comparison of 30-day mortality between transfemoral and transapical subgroups dependent on severity of mitral stenosis. MAC, mitral annular calcification; MS, mitral stenosis.
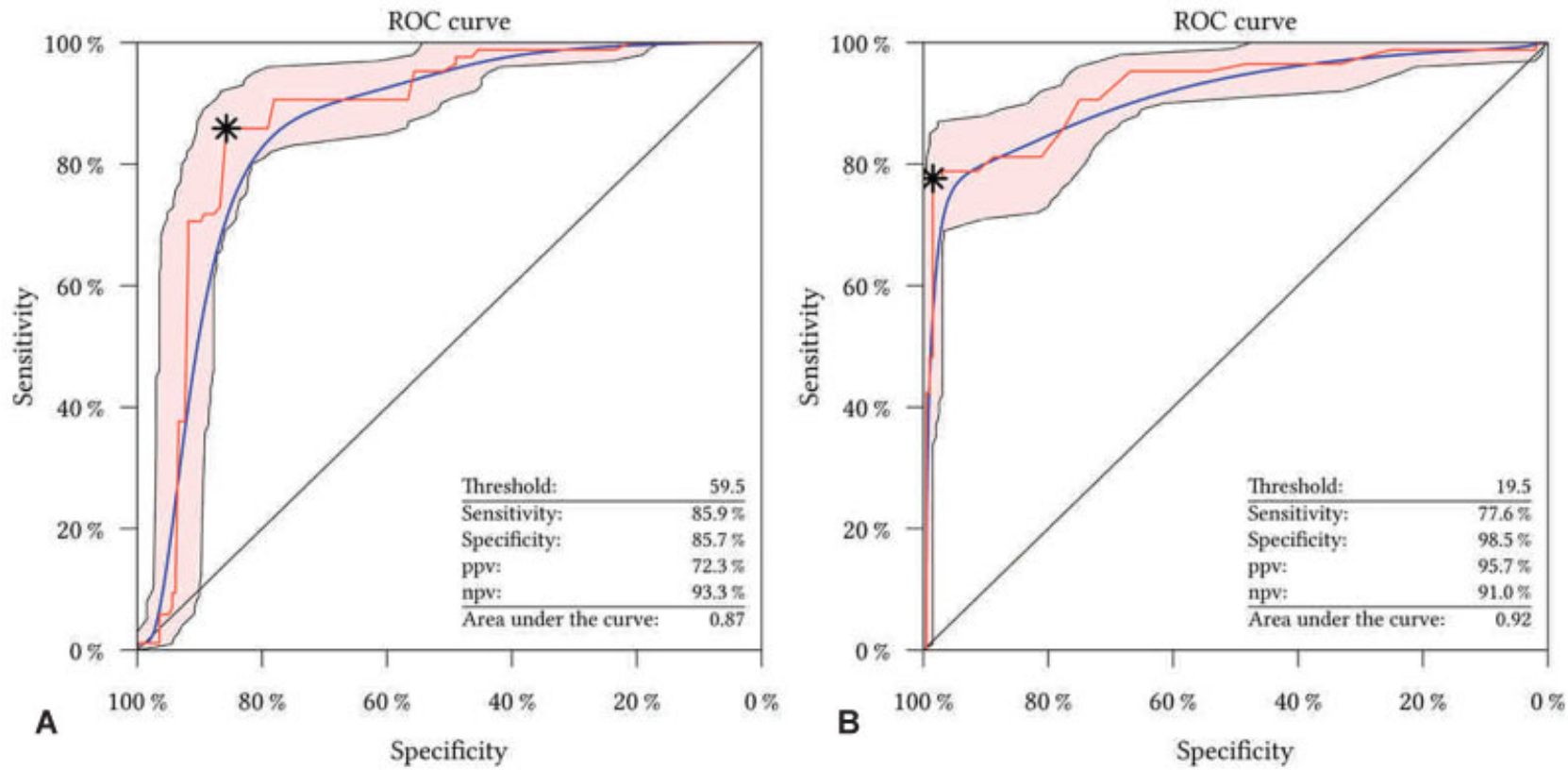

Fig. 3 ROC analysis of hemodynamic thresholds for impaired outcomes after TAVI in patients with MAC/MS. Left ventricular systolic pressure drop of $<59.5 \mathrm{~mm} \mathrm{Hg}$ (threshold) is associated with increased mortality with a positive predictive value (ppv) of $72.3 \%$ and a negative value (npv) of $93.3 \%$ (A), left ventricular end-diastolic pressure drop of $<19.5 \mathrm{~mm} \mathrm{Hg}$ (threshold) is associated with increased mortality with a ppv of $95.7 \%$ and a npv of $93.3 \%$ (B). MAC, mitral annular calcification; MS, mitral stenosis; ROC, receiver operating characteristic; TAVI, transcatheter aortic valve implantation.

impact of the TA approach on acute outcomes, although it was not shown to be an independent risk factor after TAVI in this study. ${ }^{22}$ Also, the TA access presented no negative impact on long-term survival.

In our study, first evidence for association of acute hemodynamic changes and mortality after TAVI with concomitant
MAC/MS was found. ROC analysis revealed increased LV pressure drop after THV insertion as highly predictive of 30-day mortality. Consequences of MS relief in patients with concomitant AS are well investigated. Here, elimination of MS can lead to acute LV failure caused by a sudden increase of preload on a hypertrophied LV leading to pulmonary edema. ${ }^{23,24}$ 
Table 4 Invasive hemodynamic measurements during TAVI for entire study population and subgroups

\begin{tabular}{|c|c|c|c|c|c|c|}
\hline & $\begin{array}{l}\Sigma \\
n=1058\end{array}$ & $\begin{array}{l}\text { MAC } \\
n=352\end{array}$ & $\begin{array}{l}\text { Mild MS } \\
n=528\end{array}$ & $\begin{array}{l}\text { Moderate MS } \\
n=167\end{array}$ & $\begin{array}{l}\text { Severe MS } \\
n=11\end{array}$ & $p$-Value ${ }^{a}$ \\
\hline \multicolumn{7}{|l|}{ Preoperative } \\
\hline Peak-to-peak gradient, mm Hg & $48.5 \pm 34.4$ & $45.3 \pm 24.1$ & $47.6 \pm 36.3$ & $54.2 \pm 35.6$ & $73.0 \pm 51.4$ & 0.23 \\
\hline Mean gradient, mm Hg & $44.6 \pm 21.3$ & $42.2 \pm 16.5$ & $43.7 \pm 20.6$ & $49.4 \pm 26.3$ & $62.8 \pm 38.9$ & 0.07 \\
\hline \multicolumn{7}{|l|}{ Postoperative } \\
\hline Peak-to-peak gradient, mm Hg & $4.2 \pm 6.5$ & $4.4 \pm 3.9$ & $3.8 \pm 6.0$ & $5.2 \pm 9.8$ & $4.5 \pm 5.1$ & 0.50 \\
\hline Mean gradient, $\mathrm{mm} \mathrm{Hg}$ & $11.5 \pm 7.1$ & $12.3 \pm 5.1$ & $11.0 \pm 5.3$ & $12.3 \pm 12.5$ & $11.5 \pm 6.6$ & 0.44 \\
\hline \multicolumn{7}{|l|}{$\Delta$ Pressure } \\
\hline LV systolic, $\mathrm{mm} \mathrm{Hg}$ & $39.5 \pm 32.9$ & $43.7 \pm 27.2$ & $29.2 \pm 32.6$ & $52.6 \pm 30.1$ & $62.6 \pm 15.4$ & $<0.001$ \\
\hline LV end-diastolic, $\mathrm{mm} \mathrm{Hg}$ & $5.7 \pm 13.8$ & $4.9 \pm 13.6$ & $1.7 \pm 12.0$ & $11.4 \pm 14.3$ & $12.3 \pm 14.4$ & $<0.001$ \\
\hline AO systolic, $\mathrm{mm} \mathrm{Hg}$ & $2.5 \pm 33.9$ & $4.2 \pm 32.2$ & $-9.1 \pm 28.5$ & $18.0 \pm 35.5$ & $22.1 \pm 13.2$ & $<0.001$ \\
\hline AO diastolic, $\mathrm{mm} \mathrm{Hg}$ & $5.1 \pm 21.5$ & $6.6 \pm 21.5$ & $1.0 \pm 16.0$ & $10.2 \pm 26.5$ & $10.8 \pm 30.4$ & $<0.01$ \\
\hline
\end{tabular}

Abbreviations: AO, aorta; LV, left ventricle; MAC, mitral annular calcification; MS, mitral stenosis; TAVI, transcatheter aortic valve implantation. ${ }^{a} A N O V A$ for metric variables and $\mathrm{chi}^{2}$ for categorial variables.

Characterization of hemodynamic consequences of isolated AS relief in patients with concomitant MS is scarce, since traditionally the MV is treated first or dual valve surgery is taken into consideration. ${ }^{1,2,24}$ The herein collected hemodynamic dataset suggests adverse effect of isolated AS relief in patients with MS. Persistently lowered, MS induced preload in a decompressed LV seems to negatively impact survival after TAVI that may be caused by consecutive low-output syndrome. However, these findings are mainly hypothesis generating, and effects of observed hemodynamic changes may be influenced by the high-risk profile patient population with according comorbidities/confounders.

Different to concomitant significant MR in patients undergoing TAVI, in which a reduction in MR severity can be expected in a large proportion of patients with functional

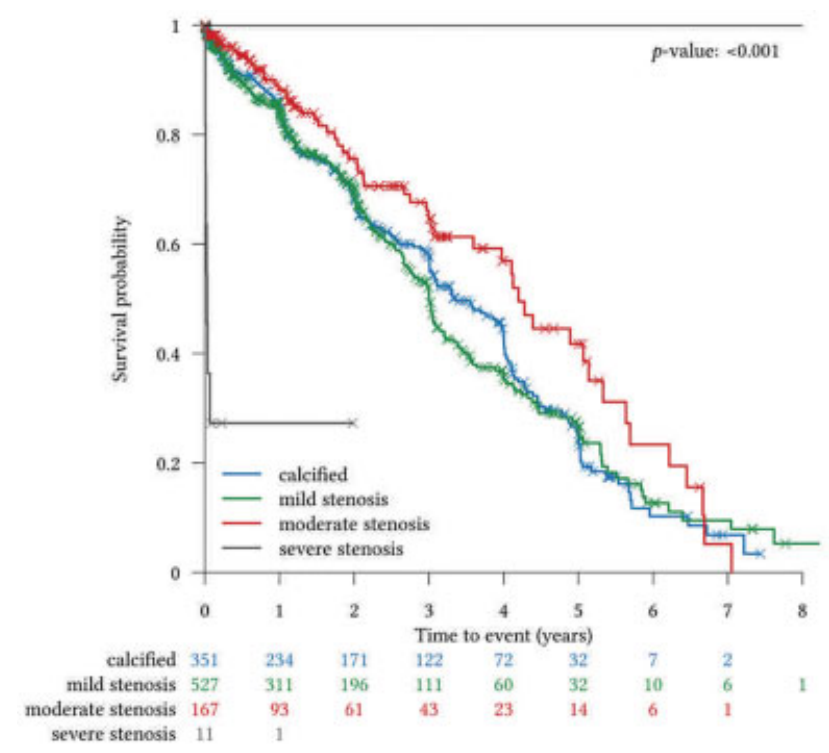

Fig. 4 Survival probability in long-term follow-up for mitral stenosis subgroups.
$M R,{ }^{25}$ patients with significant MS present a pronounced different risk profile. Due to the described severely impaired outcomes in patients with moderate and severe MS, other therapy strategies shall be commented. Traditionally, surgical combined valve replacement is the standard for concomitant valvular heart disease in low-to-intermediate risk patients. Here, a threefold higher perioperative mortality compared with isolated valve replacement is described. ${ }^{14}$ In surgery for MS alone a 30 -day mortality of $10 \%$ with a 10 -year survival of $58 \%$ in patients with pulmonary hypertension was reported. ${ }^{26}$ There are no reported outcome data for surgical treatment of high-risk patients with isolated mitral valve replacement for MS, or combined valve replacement for AS and MS. Currently, therapy of MV disease with MAC by utilization of balloon-expandable THV was described with a 30-day mortality of nearly $30 \%{ }^{27,28}$ Therefore, all therapeutic strategies for this special subset of patients seem to be associated with pronounced impaired postoperative outcomes, especially when taking the high-risk profile of the herein investigated cohort into consideration. Taking the high mortality of patients with moderate and severe MS undergoing TAVI for severe AS into account, a primary surgical or palliative strategy may be also reasonable alternatives for this special subset of patients.

\section{Study Limitations}

Limitations for a retrospective, single-center study apply: no patient was randomly assigned to specific treatment and the conclusion that concomitant MAC and/or MS in TAVI procedures predicts outcomes is limited by the heterogeneity of the study group, especially in terms of several utilized THV systems and access approaches. Hemodynamic measurements could have been influenced by variable anesthesiologic regimens. Furthermore, MAC and MS were partly diagnosed by TTE/TEE and without quantification of MAC severity. 


\section{Conclusions}

TAVI in the presence of MAC/MS is associated with acceptable acute outcomes in MAC and mild MS and should be considered high-risk procedures in patients with moderate and severe MS. First hemodynamic evidence suggests LV pressure drop after THV insertion with consecutive lowoutput syndrome as possible reason. Anticipation of this postoperative complication and respective therapy strategies, in terms of sophisticated volume and inotropic support, may contribute to improved postoperative survival rates, especially in patients with moderate and severe MS. All available therapies for this special subset of patients, especially those with a high-risk profile, are associated with increased mortality. Surgical or palliative strategies may be also reasonable alternatives for patients with moderate or severe MS. Therefore, individual therapeutic strategies and meticulous procedural planning are of paramount importance to improve outcomes.

\section{Author Contributions}

Andreas Schaefer made substantial contributions to the conception and design of the work; to the acquisition, analysis, and interpretation of data for the work; to drafting the work and revising it critically for important intellectual content; to final approval of the version to be published; and agrees to be accountable for all aspects of the work in ensuring that questions related to the accuracy or integrity of any part of the work are appropriately investigated and resolved.

Harun Sarwari made substantial contributions to the conception and design of the work; to the acquisition of data for the work; to drafting the work; to final approval of the version to be published; and agrees to be accountable for all aspects of the work in ensuring that questions related to the accuracy or integrity of any part of the work are appropriately investigated and resolved.

Niklas Schofer made substantial contributions to the conception and design of the work; to drafting the work and revising it critically for important intellectual content; to final approval of the version to be published; and agrees to be accountable for all aspects of the work in ensuring that questions related to the accuracy or integrity of any part of the work are appropriately investigated and resolved.

Yvonne Schneeberger made substantial contributions to the conception and design of the work; to drafting the work and revising it critically for important intellectual content; to final approval of the version to be published; and agrees to be accountable for all aspects of the work in ensuring that questions related to the accuracy or integrity of any part of the work are appropriately investigated and resolved.

Dirk Westermann made substantial contributions to the conception and design of the work; to drafting the work and revising it critically for important intellectual content; to final approval of the version to be published; and agrees to be accountable for all aspects of the work in ensuring that questions related to the accuracy or integrity of any part of the work are appropriately investigated and resolved.

Gerhard Schoen made substantial contributions to the conception and design of the work; to the acquisition, analysis, and interpretation of data for the work, to drafting the work and revising it critically for important intellectual content; to final approval of the version to be published; and agrees to be accountable for all aspects of the work in ensuring that questions related to the accuracy or integrity of any part of the work are appropriately investigated and resolved.

Stefan Blankenberg made substantial contributions to the conception and design of the work; to drafting the work and revising it critically for important intellectual content; to final approval of the version to be published; and agrees to be accountable for all aspects of the work in ensuring that questions related to the accuracy or integrity of any part of the work are appropriately investigated and resolved.

Hermann Reichenspurner made substantial contributions to the conception and design of the work; to drafting the work and revising it critically for important intellectual content; to final approval of the version to be published; and agrees to be accountable for all aspects of the work in ensuring that questions related to the accuracy or integrity of any part of the work are appropriately investigated and resolved.

Ulrich Schäfer made substantial contributions to the conception and design of the work; to drafting the work and revising it critically for important intellectual content; to final approval of the version to be published; and agrees to be accountable for all aspects of the work in ensuring that questions related to the accuracy or integrity of any part of the work are appropriately investigated and resolved.

Lenard Conradi made substantial contributions to the conception and design of the work; to the acquisition, analysis, and interpretation of data for the work; to drafting the work and revising it critically for important intellectual content; to final approval of the version to be published; and agrees to be accountable for all aspects of the work in ensuring that questions related to the accuracy or integrity of any part of the work are appropriately investigated and resolved.

\section{Disclosure Statement}

A. Schaefer has received travel compensation from Symetis SA and Abbott Vascular Inc.

U. Schaefer is a proctor for Symetis SA and Medtronic, is a consultant to Medtronic and Symetis SA, and has received lecture fees from Medtronic.

L. Conradi is a proctor for Boston Scientific and Medtronic and proctor and consultant for Edwards Lifesciences. All others authors have nothing to disclose.

Funding Statement

No funding was received regarding this work. 


\section{References}

1 Baumgartner H, Falk V, Bax JJ, et al; ESC Scientific Document Group. 2017 ESC/EACTS Guidelines for the management of valvular heart disease. Eur Heart J 2017;38(36):2739-2791

2 Nishimura RA, Otto CM, Bonow RO, et al. 2017 AHA/ACC Focused Update of the 2014 AHA/ACC Guideline for the management of patients with valvular heart disease: a report of the American College of Cardiology/American Heart Association Task Force on Clinical Practice Guidelines. J Am Coll Cardiol 2017;70(02):252-289

3 Leon MB, Smith CR, Mack MJ, et al; PARTNER 2 Investigators. Transcatheter or surgical aortic-valve replacement in intermediate-risk patients. N Engl J Med 2016;374(17):1609-1620

4 Reardon MJ, Van Mieghem NM, Popma JJ, et al; SURTAVI Investigators. SURTAVI Investigators. Surgical or transcatheter aorticvalve replacement in intermediate-risk patients. $\mathrm{N}$ Engl J Med 2017;376(14):1321-1331

5 Regev E, Finkelstein A, Assali A, et al. Comparison of outcome of transcatheter aortic valve implantation for severe aortic stenosis in 3 age groups ( $\leq 70 ; 71$ to 80 , and $\geq 81$ years). Am J Cardiol 2017; 120(09):1607-1611

6 Doshi R, Shlofmitz E, Meraj P. Comparison of outcomes and complications of transcatheter aortic valve implantation in women versus men (from the National Inpatient Sample). Am J Cardiol 2018;121(01):73-77

7 Anand A, Harley C, Visvanathan A, et al. The relationship between preoperative frailty and outcomes following transcatheter aortic valve implantation: a systematic review and meta-analysis. Eur Heart J Qual Care Clin Outcomes 2017;3(02):123-132

8 Levi A, Codner P, Masalha A, et al. Predictors of 1-year mortality after transcatheter aortic valve implantation in patients with and without advanced chronic kidney disease. Am J Cardiol 2017;120 (11):2025-2030

9 Schaefer A, Neumann N, Linder M, et al. Outcomes with a latest generation self-expandable, intra-annular, re-sheathable transcatheter heart valve system: analysis of patients with impaired left ventricular function and determinants for pacemaker implantation. Clin Res Cardiol 2018;107(10):914-923

10 Schofer N, Deuschl F, Schön G, et al. Comparative analysis of balloon- versus mechanically-expandable transcatheter heart valves considering landing zone calcification. J Cardiol 2018;71 (06):540-546

11 Abramowitz Y, Kazuno Y, Chakravarty T, et al. Concomitant mitral annular calcification and severe aortic stenosis: prevalence, characteristics and outcome following transcatheter aortic valve replacement. Eur Heart J 2017;38(16):1194-1203

12 Ancona MB, Giannini F, Mangieri A, et al. Impact of mitral annular calcium on outcomes after transcatheter aortic valve implantation. Am J Cardiol 2017;120(12):2233-2240

13 Nishimura RA, Vahanian A, Eleid MF, Mack MJ. Mitral valve disease-current management and future challenges. Lancet 2016;387(10025):1324-1334

14 Unger P, Lancellotti P, de Cannière D. The clinical challenge of concomitant aortic and mitral valve stenosis. Acta Cardiol 2016; 71(01):3-6
15 Carabello BA. Modern management of mitral stenosis. Circulation 2005;112(03):432-437

16 Kappetein AP, Head SJ, Généreux P, et al; Valve Academic Research Consortium (VARC)-2. Updated standardized endpoint definitions for transcatheter aortic valve implantation: the Valve Academic Research Consortium-2 consensus document (VARC-2). Eur J Cardiothorac Surg 2012;42(05):S45-S60

17 Schaefer A, Treede H, Schoen G, et al. Improving outcomes: casematched comparison of novel second-generation versus firstgeneration self-expandable transcatheter heart valves. Eur J Cardiothorac Surg 2016;50(02):368-373

18 Joseph L, Bashir M, Xiang Q et al. Prevalence and outcomes of mitral stenosis in patients undergoing transcatheter aortic valve replacement: findings from the Society of Thoracic Surgeons/ American College of Cardiology Transcatheter Valve Therapies Registry. JACC Cardiovasc Interv 2018;11(07):693-702

19 Grover FL, Vemulapalli S, Carroll JD, et al; STS/ACC TVT Registry. 2016 Annual Report of The Society of Thoracic Surgeons/American College of Cardiology Transcatheter Valve Therapy Registry. J Am Coll Cardiol 2017;69(10):1215-1230

20 Adler Y, Fink N, Spector D, Wiser I, Sagie A. Mitral annulus calcification-a window to diffuse atherosclerosis of the vascular system. Atherosclerosis 2001;155(01):1-8

21 Biancari F, Rosato S, D’Errigo P, et al; OBSERVANT Research Group. Immediate and intermediate outcome after transapical versus transfemoral transcatheter aortic valve replacement. Am J Cardiol 2016;117(02):245-251

22 Koifman E, Magalhaes M, Kiramijyan S, et al. Impact of transfemoral versus transapical access on mortality among patients with severe aortic stenosis undergoing transcatheter aortic valve replacement. Cardiovasc Revasc Med 2016;17(05): 318-321

23 Suh WM, Kern MJ. Addressing the hemodynamic dilemma of combined mitral and aortic stenosis. Catheter Cardiovasc Interv 2008;71(07):944-949

24 Unger P, Rosenhek R, Dedobbeleer C, Berrebi A, Lancellotti P. Management of multiple valve disease. Heart 2011;97(04):272277

25 Muratori M, Fusini L, Tamborini G, et al. Mitral valve regurgitation in patients undergoing TAVI: Impact of severity and etiology on clinical outcome. Int J Cardiol 2019:S0167-5273(18)36307-1

26 Yang B, DeBenedictus C, Watt T, et al. The impact of concomitant pulmonary hypertension on early and late outcomes following surgery for mitral stenosis. J Thorac Cardiovasc Surg 2016;152 (02):394-400.e1

27 Wilbring M, Alexiou K, Tugtekin SM, et al. Pushing the limitsfurther evolutions of transcatheter valve procedures in the mitral position, including valve-in-valve, valve-in-ring, and valve-in-native-ring. J Thorac Cardiovasc Surg 2014;147(01): 210-219

28 Guerrero M, Dvir D, Himbert D, et al. Transcatheter mitral valve replacement in native mitral valve disease with severe mitral annular calcification: results from the first multicenter global registry. JACC Cardiovasc Interv 2016;9(13):1361-1371 